\title{
Mortalité du sapin baumier défolié par la tordeuse des bourgeons de l'épinette dans la région de la Gatineau au Québec
}

\author{
par
}

Jean Beaulieu et Yvan J. Hardy ${ }^{1}$

\section{Résumé}

Une méthode d'analyse a été mise au point en vue de différencier la mortalité du sapin baumier attribuable à la tordeuse des bourgeons de l'épinette de la mortalité régulière, pour la région de la Gatineau au Québec. Cette méthodologie a d'abord été développée en utilisant la technique de la régression linéaire multiple et un générateur pseudo-aléatoire de distribution uniforme. À cause de problèmes de biais liés à l'estimation par la méthode des moindres carrés de la variance des paramètres de la régression lorsque la variable dépendante est binaire $(0,1)$, la méthode d'analyse probit a à son tour été employée. Dans ce cas l'estimation des paramètres et de leur variance est effectuée par la méthode du maximum de vraisemblance. Les résultats démontrent que les deux moyens mathématiques utilisés sont équivalents lorsqu'on compare les pourcentages de mortalité dûs à la tordeuse qui en découlent. Les données utilisées couvrent la période de 1958 à 1979, tandis que l'épidémie sévissait de 1968 à 1975 .

En 1979 , environ $55 \%$ des tiges avaient été tuées par la tordeuse selon les résultats obtenus à l'aide de la méthode développée, ce qui correspond à $53 \%$ du volume. L'érablière à bouleau jaune est plus affectée, en pourcentage, que la sapinière; toutefois, en valeur absolue, on ne trouve pas de différence significative. De plus, on y démontre que les pulvérisations d'insecticide chimique ont retardé l'arrivée de la mortalité, cependant cette dernière s'est aussitôt manifestée lors de l'arrêt du programme de pulvérisation.

\section{Abstract}

This paper presents a method of analysis which differentiates between spruce budworm caused mortality and regular mortality on balsam fir in the Gatineau region in Quebec. A first attempt was made using multiple linear regression and a uniform random number generator. In order to overcome the bias inherent to the least squares method when dealing with a binary $(0,1)$ dependent variable, a profit analysis was also conducted. In this case, the parameters and their variance were estimated using likehood method. These two approaches proved to be equivalent when percent budworm caused mortality was compared within the 1958 to 1979 period covered by the data at hand, while the outbreak lasted from 1968 to 1975.

In 1979 , approximately $55 \%$ of the stems had been killed by the budworm, accounting for $53 \%$ of the volume. Maple-yellow birch associations were more affected than fir associations although no significant difference was found. Fir mortality was delayed by aerial spraying of insecticides but this advantage disappeared as soon as the spray operations came to an end.

Département d'aménagement et de sylviculture, Faculté de foresterie et géodésie, Université Laval, Québec, G1K 7P4

\author{
Introduction
}

La tordeuse des bourgeons de l'épinette, Choristoneura funiferana (Clem.), un insecte défollateur du sapin baumier, Abies balsamea Mill., et des épinettes blanche et rouge, Picea glauca Voss et P. rubens Sarg., est considérée par certains comme l'ennemi numéro un au niveau économique des forêts résineuses du nord-est de l'Amérique (Miller, 1975). Des épidémies répétées, (Blais, 1965; Swaine et Craighead, 1924), et une tendance de ces dernières à revenir à intervalles plus courts (Blais, 1974) ont été à l'origine de nombreuses études sur la biologie de l'insecte, (Mitchell et Seabrook, 1970), la susceptibilité des forêts (Blais, 1968; Hardy et Dorais, 1976; Morris et Bishop, 1951) et l'épidémiologie (Blackman, 1919; Blais 1964). Toutefois, rares sont les études d'impact sur le milieu forestier (Baskerville et MacLean, 1979; Batzer, 1973; Blais 1981; McLintock, 1955), malgré de nombreux estimés imprécis (Baskerville, 1960; Blais, 1958; Morris, 1946). Au Québec, seuls Blais et McLintock ont mesuré l'impact de la tordeuse sur la production ligneuse et la présente étude doit être considérée comme une autre contribution à la résolution du problème de l'impact de cet insecte sur la production de matière ligneuse. Deux méthodes mathématiques ont été utilisées à l'intérieur d'une même méthodologie, et leurs résultats ont été comparés. Compte tenu que l'épidémie a originé en 1968 dans la région de la Gatineau pour disparaître en 1975, cette dernière fut choisie pour conduire notre étude, car il s'agissait là d'une des seules régions de la province où des données dendrométriques suivies étaient disponibles sur une période qui englobait le début et la fin de l'épidémie.

\section{Matériel}

Cette étude a été rendue possible grâce à la collaboration de la Compagnie Internationale de Papier qui a mis à la disposition des auteurs une partie de sa banque de données forestières créée à l'aide d'un inventaire forestier continuel avec remplacement partiel tel que décrit par Cunia en 1964. Les mesures ont été prises dans des parcelles-échantillons d'un dixième d'acre (1/10 a) disposées par groupes d'au plus trois (3) et réparties de façon aléatoire sur le terrain après stratification par rapport aux types de peuplements. Les premières mesures furent recueillies en 1958 et répétées environ tous les cinq (5) ans jusqu'en 1979, soit quelques années après la principale vague de l'épidémie qui se termina en 1975 dans ce secteur. De 1958 jusqu'au début de l'infestation en 1968, les parcelles-échantillons ont été mesurées à deux (2) reprises, tandis que quatre (4) remesurages sont venus s'ajouter entre 1968 et 1979 .

Les données de cent trente-six (136) parcelles-échantillons, situées dans la région de la Gatineau, figure 1, ont servi à mesurer l'ampleur des dégâts. Les peuplements forestiers qui colonisent cette zone appartiennent à deux (2) domaines climaciques (Grandtner, 1980): le premier, celui de l'érablière à bouleau jaune, compte trente-cinq (35) parcelles, le second, celui de la sapinière, est composé de deux groupements, la sapinière à bouleau jaune et la sapinière à bouleau blanc, 

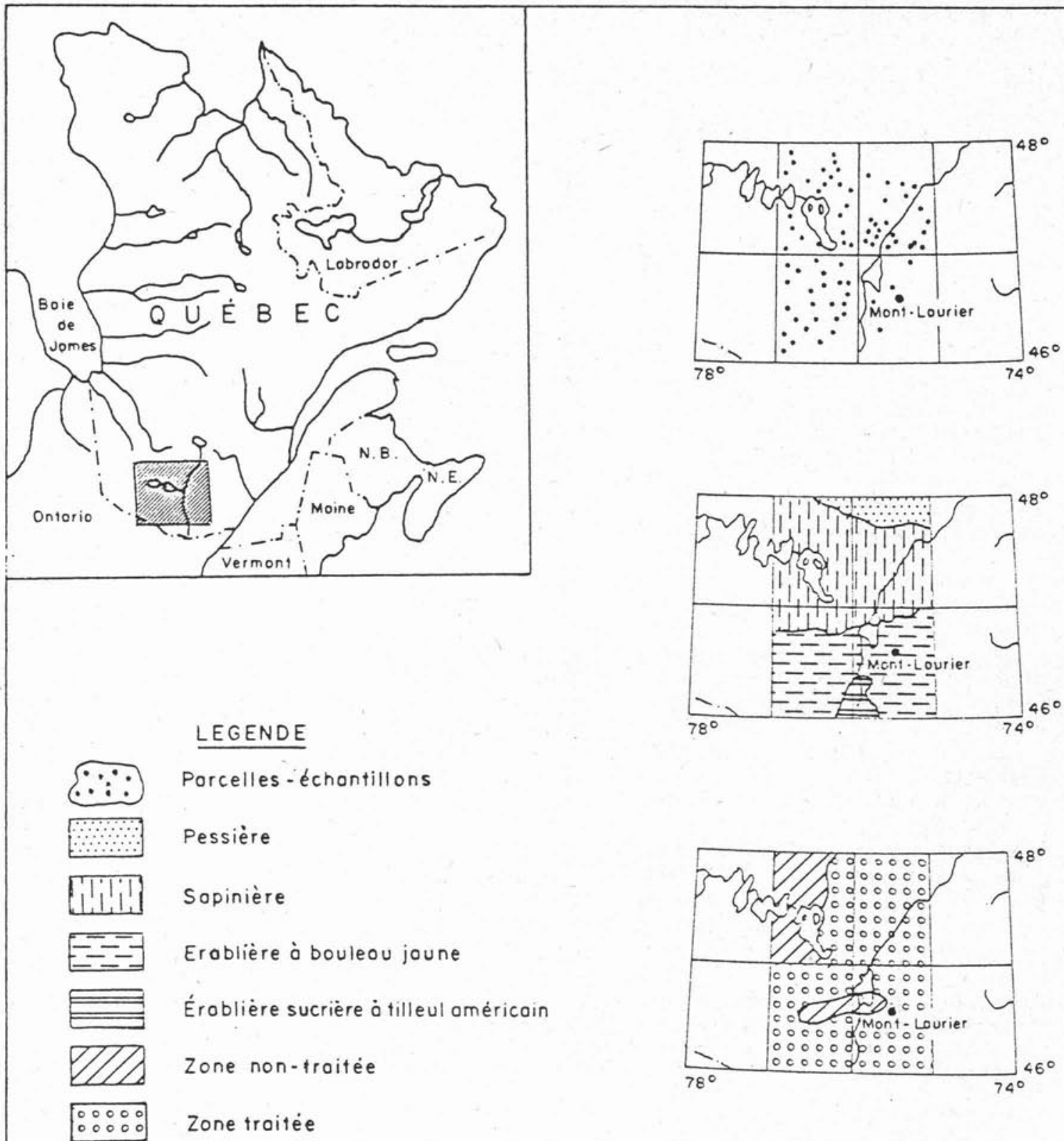

Figure 1. Localisation et caractérisation du territoire ayant servi à l'étude d'impact de la tordeuse des bourgeons de l'épinette.

comptant respectivement quatre-vingt-six (86) et quinze (15) parcelles. De plus, grâce aux inventaires de Ministère de l'Énergie et des Ressources du Québec, le territoire a pu être stratifié selon la durée et l'intensité de l'épidémie, et les interventions de contrôle qui ont eu lieu entre 1971 et 1974 , ce qui a permis d'apprécier l'effet des pulvérisations aériennes sur l'état des peuplements.

\section{Méthode}

Les premiers mesurages effectués de 1958 à 1962 ont servi d'assise à cette étude en déterminant les principaux paramètres descriptifs (essences, diamètres, étages, etc.) des arbres alors observés. Les remesurages qui ont suivi, ont pour leur part permis de déterminer l'évolution des paramètres descriptifs initiaux et ont également permis de recueillir ceux associés aux recrues. La figure 2 illustre l'évolution théorique de l'état des arbres constituant cette population. Avant le début de l'épidémie, deux (2) causes de mortalité y sont identifiées; la mortalité irrégulière due principalement au chablis, et la mortalité régulière découlant du vieillissement et de la compétition. Par la suite, lorsqu'apparaît la tordeuse des bourgeons de l'épinette, à partir de 1968, une autre cause de mortalité, celle-ci attribuable à l'insecte, vient s'ajouter aux deux autres. Toutefois, la mortalité totale, c'est-à-dire la mortalité régulière et la mortalité irrégulière due à différentes causes a été notée sans distinction.

Afin de ne pas attribuer à l'insecte la mortalité causée par la compétition et le vieillissement, il fallait mettre au point une méthode permettant d'isoler la mortalité due à la tordeuse de celle due aux autres causes. C'est ainsi que l'on a eu recours à la régression multiple et à la méthode d'analyse probit en vue de prédire 1) la mortalité régulière durant la période d'infestation, en utilisant une méthode s'apparentant à celle mise au point par Lanford (1975). Pour prédire 2) la mortalité irrégulière due au chablis, au cours de la même période, on a utilisé un générateur pseudo-aléatoire de distribution normale. 
Premier mesurage

$(1958$ - 1962)

Vivants

Deuxième mesurage

$(1963$ - 1967)

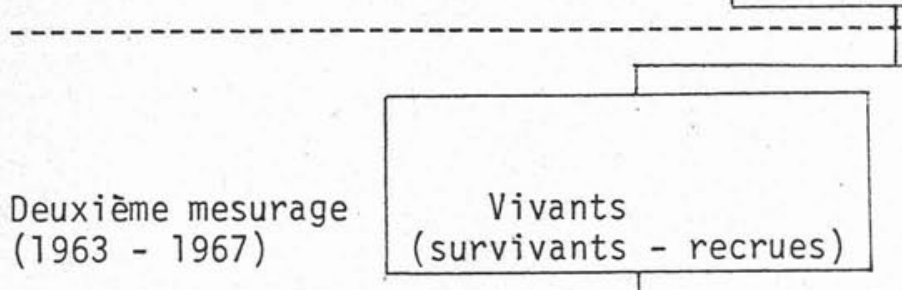

I

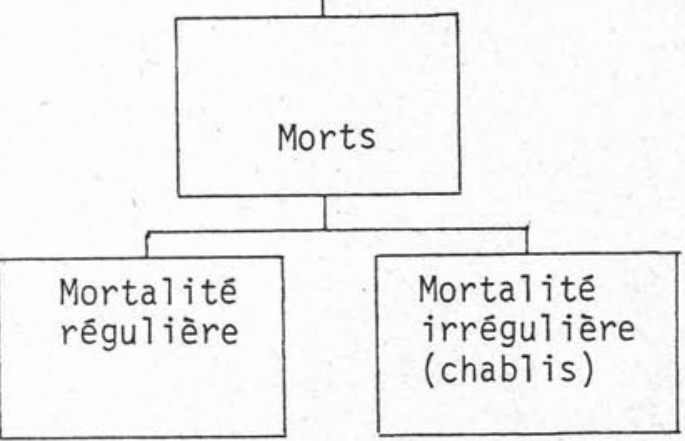

Troisième mesurage et suivants

$(1968$ - 1979)
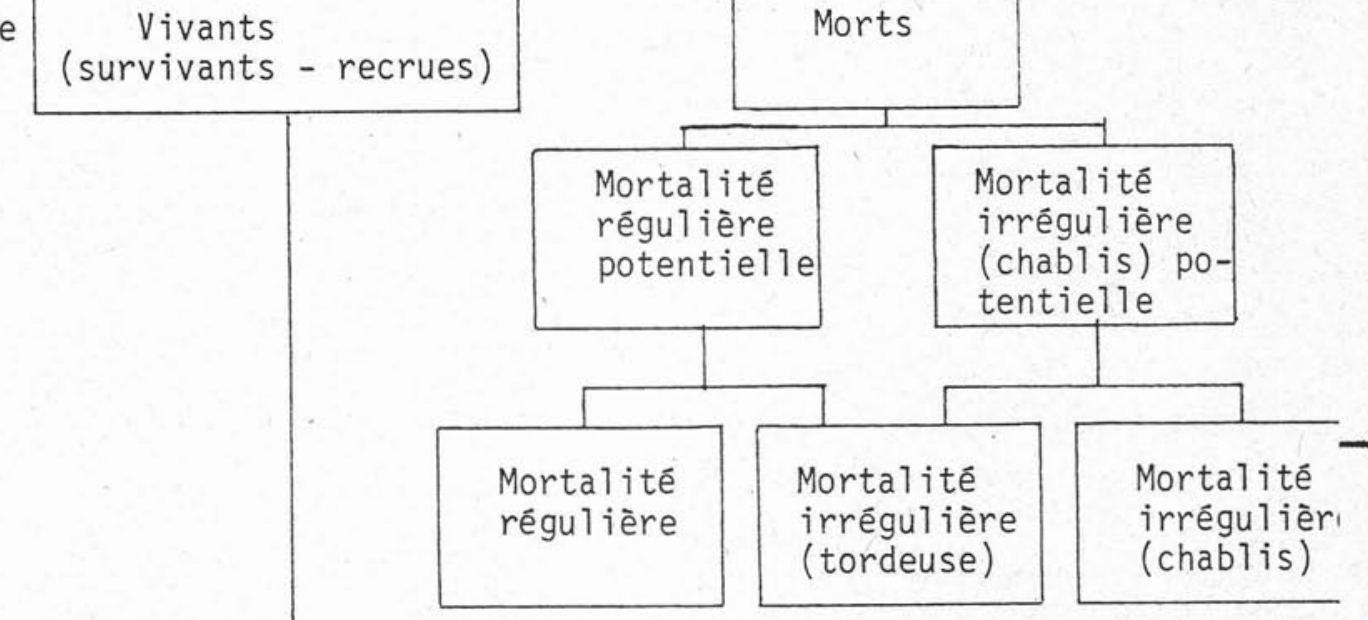

Figure 2. Schéma explicatif de la méthode utilisée pour déterminer dans le temps l'évolution de l'état des arbres.

\section{1) Prédiction de la mortalité régulière}

Le pourcentage moyen de mortalité régulière constatée entre les deux premiers mesurages a servi à déterminer la probabilité de mortalité dans les mesurages subséquents. La probabilité qu'un arbre meure de causes régulières entre deux mesurages a été déterminée à l'aide de trois (3) paramètres descriptifs, soient: le diamètre à hauteur de poitrine (DHP), le taux annuel d'accroissement en diamètre (TAD) et la classe d'étage (ETA). Compte-tenu du fait que seuls 28 sapins baumiers sur 1642 tiges présentes lors du mesurage original présentaient à la fois un état indiquant la mortalité régulière et la mesure des trois paramètres descriptifs, nous avons du recourir à la stratification afin de diminuer l'erreurtype de l'estimé. Le diamètre à hauteur de poitrine (DHP) a servi de variable de stratification. Nous avons formé quatre groupes dans lesquels étaient répartis les 28 sapins baumiers morts. Le tableau I indique comment la méthode de stratification, proposée par Dalenius et Hodges (1959) et rapportée par Cochran (1963), qui consiste en la cumulation de la racine carrée des effectifs, a servi à déterminer les limites des classes de diamètre pour chaque groupe.
Tableau I. Stratification en quatre (4) groupes des arbres morts régulièrement par rapport à leur diamètre à hauteur de poitrine.

\begin{tabular}{|c|c|c|c|c|c|}
\hline $\begin{array}{c}\text { Classes de } \\
\text { diamètres } \\
\text { (pouces) }\end{array}$ & \multicolumn{2}{|c|}{ Effectifs } & \multicolumn{3}{|c|}{$\begin{array}{l}\text { Cumulation } \\
\text { de } \sqrt{\text { effectifs }}\end{array}$} \\
\hline $\begin{array}{r}4 \\
5 \\
6 \\
7 \\
8 \\
9 \\
10 \\
11 \\
\end{array}$ & $\begin{array}{l}7 \\
8 \\
2 \\
3 \\
3 \\
3 \\
0 \\
2 \\
\end{array}$ & & & $\begin{array}{r}2.6 \\
5.4 \\
6.8 \\
8.6 \\
10.3 \\
12.0 \\
12.0 \\
13.5 \\
\end{array}$ & \\
\hline \multirow{2}{*}{ Limites des strates } & \multicolumn{2}{|c|}{ Strates } & & & \\
\hline & 1 & 2 & 3 & & 4 \\
\hline $\begin{array}{l}\text { Limite des classes } \\
\text { de diamètre (pouces) }\end{array}$ & $<4.5$ & $4.5-5.49$ & $5.5-7.49$ & $3>$ & 7.5 \\
\hline $\begin{array}{l}\text { Limites de } \\
\text { cum. } \sqrt{\text { effectifs }}\end{array}$ & 3.375 & 6.75 & 10.125 & & 13.50 \\
\hline
\end{tabular}


De là, une équation de régression multiple exprimant la probabilité de mortalité de causes régulières a été établie pour chaque groupe.

\section{Ces équations sont:}

Groupe 1: Diamètres à hauteur de poitrine inférieurs à 4.5 pouces

$\mathrm{P}=1.763107-0.002127 \times \mathrm{TAD}^{-1}-0.003178 \times$
$E T A^{2}+0.201219 \times \mathrm{DHP} \times \mathrm{TAD}^{-1 / 2}-0.402748 \times$
$\left(\mathrm{DHP} \times \mathrm{TAD}^{-1}\right)^{1 / 2}-0.446688 \times \mathrm{DHP}+0.592455 \times$
$T A D$

TAD

$R^{2}=0.34 \quad E T E=0.12766 \quad N=362$

Groupe 2: Diamètres à hauteur de poitrine supérieurs ou égaux à 4.5 pouces et inférieurs à 5.5 pouces

$P=-0.118839-0.010231 \times T^{T} D^{-1}+0.035290 \times$ $\mathrm{DHP} \times \mathrm{TAD}^{-1 / 2}+1.400054 \times \mathrm{TAD}+0.000015 \times \mathrm{TAD}^{-2}$ $-0.097311 \times \mathrm{DHP}$

$R^{2}=0.41 \quad$ ETE $=0.11249 \quad N=511$

Groupe 3: Diamètres à hauteur de poitrine supérieurs ou égaux à 5.5 pouces et inférieurs à 7.5 pouces

$P=-0.041443+0.000016 \times T^{T A D}{ }^{-2}+0.023973 \times$

$\mathrm{DHP} \times \mathrm{TAD}^{-1 / 2}-0.0693064 \times \mathrm{DHP}+1.037869 \times \mathrm{TAD}$

$R^{2}=0.33 \quad$ ETE $=0.09206 \quad \mathrm{~N}=550$

Groupe 4: Diamètres à hauteur de poitrine supérieurs ou égaux à 7.5 pouces

$P=-0.259098+0.161950 \times T^{T A D^{-1 / 2}}-0.005175 \times$

$\mathrm{ETA}^{2}-0.034096 \times\left(\mathrm{DHP} \times \mathrm{TAD}^{-1}\right)^{1 / 2}+0.014367 \times$

$\mathrm{DHP}$

$\mathrm{R}^{2}=0.48 \quad \mathrm{ETE}=0.14427 \quad \mathrm{~N}=194$

$\mathrm{P} \quad=$ Probabilité de mortalité régulière

$\mathrm{DHP}=$ Diamètre à hauteur de poitrine

$\mathrm{TAP}=$ Taux annuel d'accroissement en diamètre

$E T A=$ Étage

ETE = Écart-type de l'estimé

$\mathrm{R}^{2}=$ Coefficient de détermination

$\mathrm{N} \quad=$ Nombre d'observations

Plusieurs auteurs, qui ont étudié la mortalité dans les peuplements naturels, se sont heurtés au fait qu'elle est extrêmement variable (Ek, 1974; Hamilton, 1974; Lanford, 1975; Lee, 1971; Mouserud 1976), ce qui tend à affaiblir le coefficient de détermination des équations de prédiction. De plus, en accord avec Neter et Maynes (1970) et Hamilton (1974), nous remarquons que lorsque nous établissons une régression linéaire entre une variable dépendante binaire $(0,1)$ et une ou des variables indépendantes continues, la signification du coefficient de détermination est confuse. Ainsi, on ne peut dans ce cas, comparer la valeur du coefficient à une valeur maximale de 1 et l'interprétation usuelle ne tient plus. En effet, le coefficient de détermination ne peut atteindre la valeur 1 que si les variables indépendantes sont elles aussi binaires, soit de type 0,1 . D'autre part, lorsque la variable dépendante est binaire, l'hypothèse d'homogénéité des variances sous-jacente à l'utilisation de la méthode des moindres carrés pour estimer les paramètres de la régression est violée. Les estimateurs des paramètres sont toujours non biaisés, mais leurs variances le sont et par conséquent, la construction d'intervalles de confiance est hasardeuse et les tests d'hypothèses ne sont plus valides (Malinvaud, 1964). On peut éliminer ce problème en utilisant le modèle d'analyse probit dont les paramètres sont estimés par la méthode du maximum de vraisemblance. (Finney, 1971; Sprent, 1969). En plus, ce type de modèle permet d'éviter que des valeurs prédites soient inférieures à zéro ou supérieures à un, ce qui serait incompatible avec la notion de probabilité (Goldberger, 1964).

Le modèle d'analyse probit est couramment employé pour des essais d'insecticides. Dans l'utilisation que nous en faisons, nous avons remplacé le dosage par l'accroissement annuel en diamètre. Ainsi, par analogie avec ces essais d'insecticides, où la hausse de la concentration entraîne une augmentation dans le taux de mortalité chez l'insecte, une baisse dans le taux annuel de croissance en diamètre fait augmenter le nombre de sapins baumiers qui meurent.

Le modèle d'analyse probit trouvé avec les 1642 sapins baumiers est:

$P=1 / \sqrt{2 \pi} \int_{-\infty}^{y-5} e^{-x 2 / 2 d x}$

avec $y=2.506768+0.010035 \times x$

où $P=$ la probabilité de mortalité

$X=$ l'inverse de l'accroissement annuel en diamètre $\left(T^{-1} D^{-1}\right)$

Le coefficient de détermination est 0.33 . L'écart-type de l'estimé est quant à lui de 4.49 .

Les équations de régression multiple et le modèle d'analyse probit expriment le potentiel d'une tige à mourir de causes régulières, exprimé sur une échelle continue. Toutefois, dans la nature, de même que dans le modèle de mortalité régulière, la variable dépendànte ne peut prendre que deux valeurs, soit (0) si l'arbre est vivant et (1) si ce dernier est mort. Afin de reproduire ce phénomène aléatoire, on a eu recours à un générateur pseudo-aléatoire de distribution uniforme. Ainsi, lorsque lors d'un remesurage subséquent à 1967, c'est-à-dire après l'apparition de la tordeuse sur le territoire, un arbre possédait un code d'état indiquant qu'i était mort debout, donc de causes régulières ou suite à une défoliation par la tordeuse, sa probabilité de mortalité régulière potentielle était calculée à partir de l'équation de régression correspondant à son diamètre dans un premier temps et ensuite à l'aide du modèle d'analyse probit. Après cette valeur était comparée à une autre valeur située entre zéro (0) et un (1), cette dernière tirée du générateur pseudo aléatoire. Si la probabilité calculée était supérieure au nombre pseudo-aléatoire, l'arbre était alors classé parmi les arbres morts de causes régulières. Dans le cas contraire, où la probabilité calculée était inférieure au nombre pseudo-aléatoire, la mort de l'arbre devenait attribuable à une autre cause que la mortalité régulière et était attribuée à la tordeuse. On trouvera au tableau II quelques cas types où la mortalité a été classifiée selon cette méthode à l'aide de la régression multiple; on pourra de plus y constater que plus la probabilité de mortalité régulière calculée est faible, plus elle a de chances d'être inférieure au nombre pseudo-aléatoire et par conséquent de déterminer une mortalité attribuable à la tordeuse. Enfin, le tableau II illustre le rôle des paramètres sur l'attribution de la mortalité; c'est ainsi que l'on peut constater que deux arbres (021 et 033) ayant le même taux d'accroissement appelleront des verdicts différents selon la classe de diamètre à laquelle ils appartiennent, tandis que les chances qu'un arbre ayant un taux d'accroissement en diamètre élevé (012) soit classé parmi les arbres morts de causes régulières sont à peu près nulles. Le fait de tirer au hasard des valeurs d'un générateur aléatoire de distribution uniforme est équivalent à un échantillon aléatoire avec une probabilité proportionnelle à la mortalité régulière.

Tableau II. Exemples de classification par causes de mortalite selon les caractéristiques des sapins baumiers à l'aide de la régression multiple.

\begin{tabular}{|c|c|c|c|c|c|c|c|c|}
\hline \multirow{2}{*}{$\begin{array}{l}\text { No de } \\
\text { la par- } \\
\text { celle }\end{array}$} & \multirow[t]{2}{*}{$\begin{array}{l}\text { No de } \\
\text { l'arbre }\end{array}$} & \multirow{2}{*}{$\begin{array}{l}\text { Dia. } \\
\text { mètre } \\
\text { (po) }\end{array}$} & \multirow{2}{*}{$\begin{array}{c}\text { Accrois- } \\
\text { sement } \\
\text { en DHP } \\
\text { (po) }\end{array}$} & \multirow[t]{2}{*}{ Étage ${ }^{a}$} & \multirow[t]{2}{*}{$\begin{array}{l}\text { Proba- } \\
\text { bilité }\end{array}$} & \multirow{2}{*}{$\begin{array}{c}\text { Nombre } \\
\text { au } \\
\text { hasard }\end{array}$} & \multicolumn{2}{|c|}{$\begin{array}{l}\text { Causes de. } \\
\text { mortalité }\end{array}$} \\
\hline & & & & & & & $\begin{array}{l}\text { régu- } \\
\text { lière }\end{array}$ & $\begin{array}{l}\text { irrégu- } \\
\text { lière } \\
\text { tor- } \\
\text { deuse }\end{array}$ \\
\hline 00151 & 021 & 4.30 & 0.0025 & 1 & 0.30 & 0.75 & & * \\
\hline 00151 & 033 & 5.51 & 0.0025 & 3 & 0.54 & 0.32 & * & \\
\hline 08732 & 010 & 9.37 & 0.0025 & 4 & 0.94 & 0.18 & * & \\
\hline 36813 & 012 & 6.57 & 0.1875 & 2 & 0.00 & 0.45 & & * \\
\hline
\end{tabular}

\begin{tabular}{ll}
\hline Étage : & $1=$ dominant \\
2 & $=$ codominant \\
3 & $=$ intermédiaire \\
4 & $=$ supprimé
\end{tabular}




\section{2) Prédiction de la mortalité irrégulière due au chablis}

Dans le cas de la mortalité irrégulière due au chablis, on a posé l'hypothèse que cette dernière se manifeste de la même façon en période épidémique qu'en période endémique. Ainsi, pour déterminer la cause de cet état en période épidémique, on a fait appel cette fois-ci, à un générateur pseudo-aléatoire de distribution normale. Pour assurer son fonctionnement, le nombre de valeurs à fournir a dû être déterminé, soit le pourcentage de tiges mortes par rapport au nombre de tiges vivantes au mesurage précédent l'apparition de l'épidémie. De plus, la moyenne et l'écart-type de la variable remplacée doivent être inclus dans le générateur. II devient alors possible de comparer la nouvelle population (mesurages postérieurs à 1967) aux nombres générés et de déterminer s'il s'agit d'arbres tués par la tordeuse pour être ensuite renversés par le vent, ou tout simplement de chablis. Dans le cas qui nous préoccupe, ce sont les DHP des vingt-cing (25) sapins baumiers dont l'état au second mesurage indiquait une mortalité de cause irrégulière due au chablis qui ont servi à déterminer un diamètre moyen de 6.54 et un écart-type de 2.10 pouces. Ainsi, pour établir si la mortalité irrégulière était due uniquement au chablis ou à la tordeuse suivi d'un chablis, on a créé, à l'aide d'un générateur pseudo-aléatoire, un nombre de valeurs dont la distribution est normale et ayant une moyenne de 6.54 et un écarttype de 2.10, jusqu'à concurrence de $1.5 \%$ du nombre de sapins présents lors de la période de mesurage antérieure (25/1642). Les valeurs ainsi obtenues ont été par la suite comparées aux diamètres des arbres rapportés morts de causes irrégulières et lorsqu'il y avait concordance, l'arbre était classé comme mort à cause d'un chablis; tandis que dans le cas contraire, sa mort était attribuée à la tordeuse.

Enfin, deux autres classes de perte ont dû être créées: la première regroupant les arbres qui ont été abattus, la seconde appelée mortalité due à des causes non déterminables réunissant ceux dont les caractéristiques permettant de déterminer la cause de la mortalité. n'étaient pas toutes connues.

\section{Résultats}

Une comparaison systématique des résultats obtenus en utilisant la régression multiple et l'analyse probit, à l'aide de tests de différence de ratios nous a permis de découvrir que les méthodes conduisaient à des résultats équivalents. Ainsi l'impact de la présente épidémie sur le sapin baumier peut être apprécié au tableau III ,où on constate que sur les 325 pieds cubes de volume moyen à l'acre vivant en 1960, il n'en reste plus que 212 en 1979, soit une perte réelle moyenne, pour l'ensemble du territoire, de 113 pieds cubes à l'acre. Cependant, si on considère le volume maximum de 708 pieds cubes de bois produits durant cette période, on s'aperçoit que le volume vivant de 212 pieds cubes atteint seulement $30 \%$ de cette valeur. Les pertes attribuables à la tordeuse seraient d'environ 379 pieds cubes; 117 pieds cubes auraient été perdus pour toutes autres causes. En 1960, on observait la présence de 137.5 sapins baumiers à l'acre. De ce nombre, en 1979, 80.1 dans le cas de la prédiction à l'aide de la régression linéaire et 83.4 dàns le cas de celle faite en utilisant l'analyse probit étaient déclarés morts de façon irrégulière à cause de la tordeuse. Ces nombres représentent des pertes respectives de $58 \%$ et $61 \%$ des tiges. Entre 1960 et 1979 , des recrues se sont ajoutées aux arbres initiaux pour porter le potentiel de sapins baumiers à l'acre à 222.4. En 1979 , les deux méthodes permettaient d'en classer respectivement $56.5 \%$ et $53.6 \%$ dans le groupe des arbres morts de façon irrégulière à cause de la tordeuse et $11.8 \%$ et $14.8 \%$ dans celui des arbres morts de toute autre cause. On peut voir, en consultant les chiffres du tableau IV, que ce sont les

Tableau IV. Évolution de la table de peuplement des sapins baumiers vivants (nombre de tiges/acre)

\begin{tabular}{|c|c|c|c|c|c|c|}
\hline \multirow{2}{*}{$\begin{array}{l}\text { Diamètre } \\
\text { (po) }\end{array}$} & \multicolumn{4}{|c|}{ Année } & \multirow[b]{2}{*}{1977} & \multirow[b]{2}{*}{1979} \\
\hline & 1960 & 1965 & 1970 & 1975 & & \\
\hline 3 & 0.1 & & & & & \\
\hline 4 & 56.0 & 61.2 & 62.9 & 23.1 & 31.8 & 22.6 \\
\hline 5 & 35.4 & 41.0 & 43.4 & 24.2 & 24.0 & 17.3 \\
\hline 6 & 22.2 & 26.3 & 29.2 & 19.3 & 16.8 & 11.7 \\
\hline 7 & 10.7 & 15.3 & L17.6 & -12.8 & 11.5 & $-7.1]$ \\
\hline 8 & 6.3 & 7.6 & $8 . \overline{5}$ & $\overline{9} . \overline{0}$ & 8.0 & 5.4 \\
\hline 9 & 4.0 & 3.8 & 4.2 & 5.2 & 5.1 & 4.0 \\
\hline 10 & 1.6 & 1.8 & 1.8 & 1.8 & 1.8 & 1.0 \\
\hline 11 & 0.4 & 1.1 & 1.2 & 1.2 & 1.0 & 0.7 \\
\hline 12 & 0.4 & 0.2 & 0.2 & 0.5 & 0.7 & 0.4 \\
\hline 13 & 0.2 & 0.1 & 0.3 & 0.1 & 0.1 & 0.1 \\
\hline 14 & 0.2 & 0.1 & 0.1 & & & \\
\hline 15 & & & & 0.1 & & \\
\hline 16 & 0.1 & & & & & \\
\hline Total: & 137.5 & 158.5 & 169.4 & 97.3 & 100.8 & 70.3 \\
\hline $\begin{array}{l}\text { Diamètre } \\
\text { moyen }\end{array}$ & 5.28 & 5.33 & 5.38 & 5.94 & 5.73 & 5.69 \\
\hline
\end{tabular}

Tableau III. Impact de la tordeuse des bourgeons de l'épinette sur l'évolution du volume (pi ${ }^{3}$ ) moyen à l'acre du sapin baumier a) Prédiction à l'aide de la régression multiple

\begin{tabular}{|c|c|c|c|c|c|c|}
\hline Année & Vivant & $\begin{array}{l}\text { Mort régulière } \\
\text { et irrégulière } \\
\text { (chablis) }\end{array}$ & $\begin{array}{l}\text { Mort irrégu- } \\
\text { lière (tor- } \\
\text { deuse }\end{array}$ & Abattu & $\begin{array}{l}\text { Mort non-déter- } \\
\text { minable }\end{array}$ & $\begin{array}{c}\text { Total } \\
\text { potentiel } \\
\text { (sans perte) }\end{array}$ \\
\hline 60 & 324.73 & - & & - & - & 324.73 \\
\hline 65 & 378.30 & 43.99 & & 7.72 & - & 430.01 \\
\hline 70 & 419.51 & 46.49 & 1.98 & 9.57 & - & 477.55 \\
\hline 75 & 320.45 & 74.52 & 243.16 & 13.38 & $8.39^{\prime}$ & 659.90 \\
\hline 77 & 299.85 & 84.37 & 282.32 & 13.49 & 8.39 & 688.42 \\
\hline 79 & 212.35 & 93.40 & 379.14 & 14.27 & 8.77 & 707.93 \\
\hline
\end{tabular}

b) Prédiction à l'aide de la méthode d'analyse probit

\begin{tabular}{|c|c|c|c|c|c|c|}
\hline Année & Vivant & $\begin{array}{l}\text { Mort régulière } \\
\text { et irrégulière } \\
\text { (chablis) }\end{array}$ & $\begin{array}{l}\text { Mort irrégu- } \\
\text { lière (tor- } \\
\text { deuse }\end{array}$ & Abattu & $\begin{array}{l}\text { Mort non-déter- } \\
\text { minable }\end{array}$ & $\begin{array}{c}\text { Total } \\
\text { potentiel } \\
\text { (sans perte) }\end{array}$ \\
\hline 60 & 324.73 & - & & - & - & 324.73 \\
\hline 65 & 378.30 & 43.99 & & 7.72 & - & 430.01 \\
\hline 70 & 419.51 & 47.09 & 1.38 & 9.57 & - & 477.55 \\
\hline 75 & 320.45 & 80.90 & 244.97 & 13.38 & 0.20 & 659.90 \\
\hline 77 & 299.85 & 91.38 & 283.50 & 13.49 & 0.20 & 688.42 \\
\hline 79 & 212.35 & 101.08 & 378.42 & 14.27 & 0.20 & 707.93 \\
\hline
\end{tabular}


Tableau V. Évolution du volume moyen $\left(\mathrm{pi}^{3}\right)$ à l'acre du sapin baumier par domaine climacique.

a) Prédiction à l'aide de la régression multiple

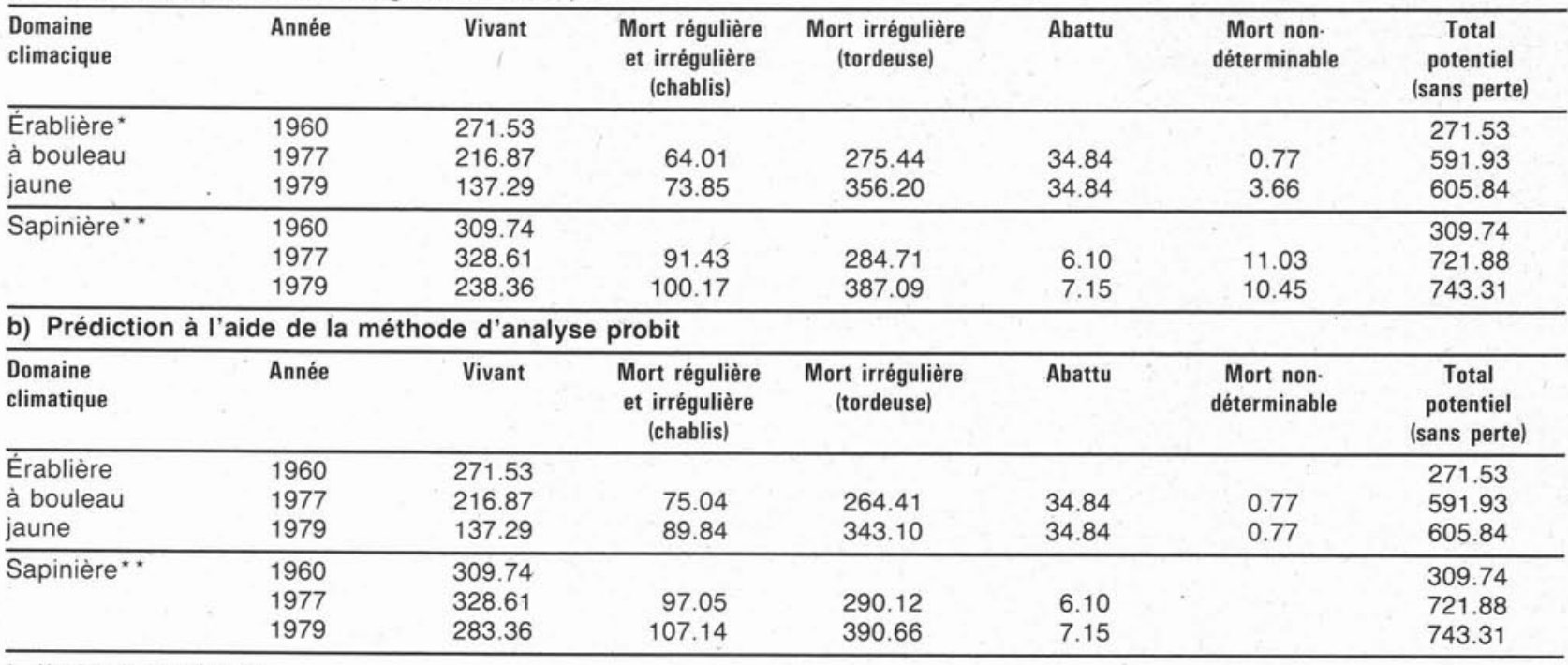

Nombre de parcelles: 35

Nombre de parcelles: 101

tiges de faible diamètre qui ont le plus subi l'effet de la défoliation. Malgré une diminution du nombre de tiges à l'acre, le diamètre moyen est passé de 5.28 pouces en 1960 à 5.69 pouces en 1979. Par contre, la mortalité régulière et la mortalité irrégulière dûe au chablis ont affecté indistinctement toutes les classes de diamètres.

Lorsque les résultats sont stratifiés par rapport aux domaines climaciques (tableau $\mathrm{V}$ ), on note rapidement que quelque soit la méthode de prédiction employée, l'érablière à bouleau jaune est le domaine le plus affecté avec des pourcentages respectifs globaux de mortalité classée comme dûe à la tordeuse en volume de $58.8 \%$, soit $356.20 / 605.84$, et de $56.5 \%$, soit $343.10 / 605.84$. Dans la sapinière on compte un pourcentage de $52.1 \%$ dans le premier cas, soit $387.09 / 743.31$, et de $52.5 \%$ dans le second en 1979 , soit 390.66/743.31. Cependant, en appliquant un test de différence de ratios, on ne trouve pas de différence significative entre les deux domaines. Par contre, en valeur absolue, le volume des sapins baumiers morts est supérieur dans la sapinière.

Lorsque la sapinière est stratifiée en sapinière à bouleau jaune et en sapinière à bouleau blanc, on arrive à la conclusion que le sapin baumier est moins vulnérable dans le second type de peuplement avec des pourcentages respectifs, selon la méthode de prédiction utilisant la régression multiple ou la probit, de $29.2 \%$ et $29.3 \%$ de mortalité classée comme étant dûe à la tordeuse comparativement à $58.5 \%$ et $59.1 \%$ dans la sapinière à bouleau jaune. Cependant, la valeur des résultats observés dans la sapinière à bouleau blanc est hypothéquée par le faible nombre de parcelleséchantillons qu'on y trouve, soit quinze (15).

En examinant une table de contingence construite à partir du nombre d'années noté avant l'apparition de la mortalité et la variable traitée/non-traitée, et en comparant les statistiques du $X^{2}$ calculé avec la valeur théorique, on s'aperçoit qu'il y a dépendance entre les deux variantes de stratification. Le nombre d'années avant l'apparition de la mortalité tend à être inférieur ou égal à quatre (4) pour les parcelleséchantillons sur lesquelles il n'y a pas eu d'épandage d'insecticide chimique, tandis que dans le cas contraire, la majorité des parcelles-échantillons se situe sur un territoire où l'on découvre la présence de la mortalité six (6) ans après le début de l'infestation. Dans le territoire traité à l'insecticide chimique, la mortalité dûe à la tordeuse déduite à l'aide de la régression linéaire multiple s'élève à $52 \%$ comparativement à $61 \%$ dans le territoire non-traité. Lorsque le modèle d'analyse probit est utilisé à la place de la régression, les pourcentages sont respectivement $51.7 \%$ et $62.1 \%$ en 1979 . Un test de différence de ratios du volume mort sur le volume vivant potentiel en 1979, en utilisant l'ensemble des 136 places-échantillons regroupées en traitées et en non-traitées, indique que l'effet global de l'insecticide a été nul.

\section{Discussion et Conclusion}

Cette étude a été effectuée à l'aide de données forestières provenant d'une région voisine de celle étudiée par McLintock lors de la précédente épidémie. Les résultats ont révélé qu'environ $55 \%$ des sapins baumiers, qui auraient pû être potentiellement vivants à la fin sont morts, et que $60 \%$ des tiges vivantes et présentes en 1960 font partie des sapins classés comme morts à cause de la tordeuse en 1979; aussi, le pourcentage de mortalité serait inférieur à celui de $79 \%$ publié par McLintock en 1955, quoique d'un ordre de grandeur aussi désastreux. Le pourcentage moyen observé est plus près des résultats de $64 \%$ fourni par Baskerville et McLean (1979) pour le Nouveau-Brunswick, et de $56 \%$ présenté par Batzer (1973) pour le Minnesota.

D'autre part, on a démontré que les pulvérisations d'insecticides ont retardé, du moins pour un certain temps, l'arrivée de la mortalité chez le sapin baumier; cette dernière s'est toutefois manifestée avec la même intensité aussitôt que le programme de pulvérisation a été interrompu. Ces remarques ne s'appliquent cependant pas au domaine de l'érablière à bouleau jaune où les pulvérisations n'ont pas été effectuées dans des conditions optimales.

De plus, ce sont les arbres dont le diamètre était inférieur au diamètre moyen des peuplements étudiés qui ont subi la plus forte mortalité, à l'instar des peuplements étudiés par Baskerville et McLean (1979). Par conséquent, cet état de fait conduit à une situation où le diamètre moyen des arbres survivants peut augmenter sans même qu'il y ait d'accroissement. Finalement, on peut conclure que le pourcentage de mortalité, dûe à la tordeuse des bourgeons de l'épinette, observé lors de la présente épidémie est élevé et du même ordre de grandeur que celui de la précédente et en présence de tels résultats, on peut croire que cet insecte défoliateur doit toujours être considéré comme l'un des pires ennemis des forêts de conifères du Québec. Dans la section traitant des résultats, on a pu constater que quelque soit le modèle utilisé pour prédire la mortalité régulière, les taux de mortalité irrégulière dûe à la tordeuse qu'on en déduit sont équi- 
valents, Ainsi, en accord avec Cunia (1973), nous remarquons que lorsque seuls les estimés des coefficients de la régression et les écarts-types des erreurs sont requis, les résultats demeurent vrais pour n'importe laquelle des distributions. Ainsi, lorsqu'on désire prédire de façon ponctuelle un pourcentage de mortalité, on peut utiliser la régression linéaire multiple, même si dans ce cas, une hypothèse est violée, car l'estimation des paramètres est toujours sans biais. Par contre, lorsqu'on souhaite connaître en plus l'étendue de l'intervalle de confiance, la méthode d'analyse probit s'avère être celle qui doit être utilisée.

La méthodologie développée au cours de cette étude devrait être appliquée à d'autres régions où des données de base compatibles existent.

\section{Remerciements}

Les auteurs adressent leurs remerciements les plus sincères à la Compagnie Internationale de Papier, et plus particulièrement à Messieurs Gilbert Paillé et Laurent Létourneau qui nous ont facilité l'accés aux données et ont bien voulu réviser le manuscrit. Nous sommes également redevables à Messieurs Louis Hamel et Richard Mathieu, du Fonds de Recherches Forestières de l'Université Laval, qui ont largement contribué à la mise en marche de cette étude. Enfin, Messieurs René Martineau, Chhun-Huor Ung et Jacques Bégin ont également offert leurs commentaires lors de la révision du manuscrit.

\section{Bibliographie}

Batzer, H.O. 1973. Net effect of spruce budworm defoliation on mortality and growth of balsam fir. J. For. 71(1): 34-37

Blackman, M.W. 1919. The spruce budworm. Me. For. Dept. $10 \mathrm{p}$

Blais, J.R. 1958. The vulnerability of balsam fir to spruce budworm attack in northwestern Ontario, with special reference to the physiological age of the tree. For. Chron. 34(4): 405-422.

Blais, J.R. 1964. Account of a recent spruce budworm outbreak in the Laurentide Park region of Quebec and measures for reducing damage in future outbreaks (Choristoneura fumiferana). For. Chron. 40(3): 313-323.

Blais, J.R. 1965. Spruce budworm outbreaks in the past three centuries in the Laurentide Park, Quebec. For. Sci. 11(2): 130-138.

Blais, J.R. 1968. Regional variation in susceptibility of eastern North American forest to budworm attack based on history of outbreaks. For Chron. 44(3): 17-23.

Blais, J.R. 1974. The policy of keeping trees alive via spray operations may hasten the recurrence of spruce budworm. For Chron. 50(1): 19-21.

Blais, J.R. 1981. Mortality of balsam fir and white spruce following a spruce budworm outbreak in the Ottawa River watershed in Quebec. Can. J. For. Res. 11(3): 620-629

Baskerville, G.L. 1960. Mortality in immature Balsam Fir following severe budworm defoliation. For. Chron. 36(4): 342-345

Baskerville, G.L. and D.A. MacLean 1979. Budworm - caused mortality and 20-year recovery in immature Balsam Fir stands. Can. For. Serv. Mar. For. Res. Cen. Inf. Rep. M-X 102. 23 p

Cochran, W.G. 1963. Sampling techniques. J. Wiley and Sons, inc. $413 p$.

Cunia, T. 1964. What is sampling with partial replacement and why use it in continuous forest inventory. Soc. Amer. For. Proc 1964: 207-211

Cunia, T. 1973. Dummy variables and some of their uses in regression analysis. Paper, IUFRO Subjects Group S4.02, Forest Resources Inventory, Nancy, France.

Dalenius, T., and J.L. Jr. Hodges, 1959. Minimum variance stratification. J-Amer. Strat. Assoc. 54: 88-101.

Ek, A.R. 1974. Nonlinear models for stand table projection in northern hardwood stands. Can. J. For. Res. 4, 23-27.

Finney, D.J. 1971. Probit analysis, 3rd ed. Cambridge University Press. 333 p

Goldberger, A.S. 1964. Econometric theory. John Wiley \& Sons Inc. New-York, $399 \mathrm{p}$

Grandtner, J.M. 1980. Écologie des forêts du Québec. Faculté de Foresterie et Géodésie, Université Laval. p. 21

Hamilton, D.A. Jr. 1974. Event probabilities estimated by regression. USDA Forest Service Res. Pap. INT-152, $18 \mathrm{p}$.
Hardy, Y.J., et L.G. Dorais, 1976. Cartographie du risque de retrouver de la mortalité dans les forêts de sapin baumier attaquées par la tordeuse des bourgeons de l'épinette. J. Can. Res. For. 6(3): 262-267.

Lawford, B.L. 1975. Projection of forest stand tables based on successive measurements of permanent plots. State University of New-York, College of Environment Science and Forestry. Ph.D. thesis. $437 p$

Lee, Y.J. 1971. Predicting mortality for even-aged stands of lodgepole pine. For. Chron. 47: 29-32.

Malinvaud, E. 1964. Méthodes statistiques de l'économétrie. Dunod $634 \mathrm{pp}$.

McLintock, T.F. 1955. How damage to balsam fir develops after a spruce budworm epidemic. USDA For. Serv. Sta. Pap. Ntheast. For. Exp. Sta. $75.18 \mathrm{p}$

Miller, C.A. 1975. Spruce budworm: how it lives and what it does. For Chron. 51(4): 135-138

Mitchell, B.K., and W.D. Seabrook, 1970. Pterothoracic musculature of the adult male spruce budworm, Choristoneura fumiferana. Can. Entomol. 102(5): 627-636.

Monserud, R.A. 1976. Simulation of forest tree mortality. Forest Sci. 22: $438-444$.

Morris, R.F. 1946. Green River project. Bi-mon. Rep. For. Insect Invest. Dept. Agric. Can. 2(3): 2.

Morris, R.F., and R.L. Bishop 1951. A method of rapid forest survey for mapping vulnerability to spruce budworm damage. For. Chron. 27(2): 171-178.

Neter, J., and E.S. Maynes 1970. On the appropriateness of the correlation coefficient with a 0,1 dependent variable. J. Am. Stat. Assoc. 65(330): 501-509

Sprent, P. 1969. Models in regression and related topics. Willmer Brothers Limited. Birkenhead. $173 p$

Swaine, J.M., and F.C. Craighead, 1924. Studies on the spruce budworm (Cacoecia fumiferama Clem.) Part I. A general account of the outbreaks, injury and associated insects. Can Dept. Agric. Bull. N.S. 37: 1-27

\section{THE UNIVERSITY OF BRITISH COLUMBIA}

The University of British Columbia invites applica. tions and nominations for the position of DEAN OF THE FACULTY OF FORESTRY. The appointment will be effective July 1, 1983 or as soon as possible thereafter and is for a term of 6 years renewable at the pleasure of the Board of Governors on the recommendation of the President.

The Dean is responsible for giving academic leadership to, and administering a Faculty with three departments and thirty-five fulltime faculty members. The departments of Harvesting and Wood Science. Forest Sciences, and Forest Resources Management are responsible for the education of about 375 undergraduate and 75 graduate students in both Masters and Doctoral programs. The University owns a Research Forest of 5,000 hectares some 60 kilometers distant from the campus. It is an integral part of teaching and research.

The University is seeking candidates with administrative experience who have established reputations appropriate to Forestry research and education. and professional practice.

The University offers equal opportunity for employment to qualified male and female candidates.

In accordance with Canadian Immigration requirements the advertisement is directed to Canadian Citizens and Permanent Residents.

The closing date for applications is December 31 . 1982 or until the position is filled.

Reply to:

Dr. J. K. Stager, Chairman

President's Search Committee - Dean of Forestry Buchanan 130

The University of British Columbia

1866 Main Mall

Vancouver, B.C.

V6T 1W5 